\title{
Timing of PSR J2055+3829, an eclipsing black widow pulsar discovered with the Nançay Radio Telescope
}

\author{
L. Guillemot ${ }^{1,2}$, F. Octau ${ }^{1,2}$, I. Cognard ${ }^{1,2}$, G. Desvignes ${ }^{3}$, P. C. C. Freire ${ }^{3}$, D. A. Smith ${ }^{4}$, \\ G. Theureau ${ }^{1,2,5}$, and T. H. Burnett ${ }^{6}$
}

\author{
${ }^{1}$ Laboratoire de Physique et Chimie de l'Environnement et de l'Espace - Université d'Orléans/CNRS, 45071 Orléans Cedex 02, \\ France \\ e-mail: lucas.guillemot@cnrs-orleans.fr \\ 2 Station de radioastronomie de Nançay, Observatoire de Paris, CNRS/INSU, 18330 Nançay, France \\ 3 Max-Planck-Institut für Radioastronomie, Auf dem Hügel 69, 53121 Bonn, Germany \\ ${ }^{4}$ Centre d'Études Nucléaires de Bordeaux Gradignan, IN2P3/CNRS, Université Bordeaux 1, BP120, 33175 Gradignan Cedex, \\ France \\ 5 LUTH, Observatoire de Paris, PSL Research University, CNRS, Université Paris Diderot, Sorbonne Paris Cité, 92195 Meudon, \\ France \\ ${ }^{6}$ Department of Physics, University of Washington, Seattle, WA 98195-1560, USA
}

Received 4 June 2019 / Accepted 23 July 2019

\begin{abstract}
We report on the timing observations of the millisecond pulsar PSR J2055+3829 originally discovered as part of the SPAN512 survey conducted with the Nançay Radio Telescope. The pulsar has a rotational period of $2.089 \mathrm{~ms}$ and is in a tight $3.1 \mathrm{~h}$ orbit around a very low mass $\left(0.023 \leq m_{\mathrm{c}} \lesssim 0.053 M_{\odot}, 90 \%\right.$ c.l.) companion. Our $1.4 \mathrm{GHz}$ observations reveal the presence of eclipses of the radio signal of the pulsar, caused by the outflow of material from the companion, for a few minutes around superior conjunction of the pulsar. The very low companion mass, the observation of radio eclipses, and the detection of time variations of the orbital period establish PSR J2055+3829 as a "black widow" (BW) pulsar. Inspection of the radio signal from the pulsar during ingress and egress phases shows that the eclipses in PSR J2055+3829 are asymmetric and variable, as is commonly observed in other similar systems. More generally, the orbital properties of the new pulsar are found to be very similar to those of other known eclipsing BW pulsars. No gamma-ray source has been detected at the location of the pulsar in recent Fermi-LAT source catalogs. We used the timing ephemeris to search ten years of Fermi Large Area Telescope (LAT) data for gamma-ray pulsations, but were unable to detect any. This nondetection could be a consequence of the large distance of the pulsar compared to those of known gamma-ray millisecond pulsars outside of globular clusters. We finally compared the mass functions of eclipsing and non-eclipsing BW pulsars and confirmed previous findings that eclipsing BWs have higher mass functions than their non-eclipsing counterparts. Larger inclinations could explain the higher mass functions of eclipsing BWs. On the other hand, the mass function distributions of Galactic disk and globular cluster BWs appear to be consistent, suggesting, despite the very different environments, the existence of common mechanisms taking place in the last stages of evolution of BWs.
\end{abstract}

Key words. pulsars: general - pulsars: individual: PSR J2055+3829 - binaries: eclipsing

\section{Introduction}

Millisecond pulsars (MSPs) are rotation-powered neutron stars with very short rotational periods $(P \lesssim 30 \mathrm{~ms})$, which are believed to have been spun-up by the accretion of matter and thus the transfer of angular momentum from a binary companion (Bisnovatyi-Kogan \& Komberg 1974; Alpar et al. 1982). While most known MSPs reside in binary systems around He or CO-white dwarf companions, a fraction of these objects are in tight binaries (with orbital periods $P_{b} \lesssim 1$ day) with very light companions $\left(m_{\mathrm{c}} \lesssim 0.05 M_{\odot}\right)$. In these "black widow" (BW) systems, the tidally locked companion star is irradiated by the pulsar's intense plasma wind. Outflowing material from the companion causes long eclipses of the pulsar's radio pulses (of $\sim 5-10 \%$ of the orbital period, typically), which are observed when the companion passes between the observer and the pulsar, provided the orbital inclination of the binary system is high enough. The exact mechanism under which the radio emission from the pulsar is eclipsed is currently unknown (see discussions in Thompson et al. 1994; Wadiasingh et al. 2017; Polzin et al. 2018). However, it is clear that eclipsing MSPs are invaluable laboratories of plasma physics (see, e.g., Main et al. 2018, for a recent example), and BW systems may also represent a formation channel for isolated MSPs (Ruderman et al. 1989). Nevertheless, although three decades have passed since the discovery of the original BW pulsar B1957+20 (Fruchter et al. 1988), and despite the recent discoveries of many BW pulsars in Fermi-LAT unassociated sources through radio observations (e.g., Ray et al. 2012; Bhattacharyya et al. 2013; Cromartie et al. 2016), the known population of BW pulsars observed to exhibit radio eclipses remains small (see Sect. 4). Discovering and studying more of these eclipsing systems is thus of prime importance.

The Nançay Radio Telescope (NRT) is a meridian telescope equivalent to a $94 \mathrm{~m}$ parabolic dish located near Orléans (France). Owing to its design, the NRT can track objects with 
declinations $\delta>-39^{\circ}$ for approximately one hour around culmination, and is thus well suited for the long-term timing of pulsars, for example, for the study of individual objects (see, e.g., Cognard et al. 2017; Octau et al. 2018, for recent examples) or for searching low-frequency gravitational waves from supermassive black hole binaries, using pulsar timing arrays (PTAs; see, e.g., Desvignes et al. 2016). With the goal of identifying new exotic pulsar systems or highly stable MSPs suitable for PTA studies, the SPAN512 pulsar survey (Desvignes et al. 2013; Octau et al. 2016; Desvignes et al., in prep.) was conducted between 2012 and 2018 at the NRT. As part of this survey, new pulsars were searched for at intermediate Galactic latitudes $\left(3.5^{\circ}<|b|<5^{\circ}\right)$ and away from the inner Galaxy (Galactic longitudes $74^{\circ}<l<150^{\circ}$ ). Observations were conducted at $1.4 \mathrm{GHz}$ with $0.5 \mathrm{MHz}$ frequency channels over a total bandwidth of $512 \mathrm{MHz}$ and a fine time resolution of $64 \mu \mathrm{s}$, to be sensitive to faint and distant MSPs. We used PRESTO pulsar searching routines (Ransom et al. 2002) to search the data for pulsars with dispersion measures (DMs) up to $1800 \mathrm{pc} \mathrm{cm}^{-3}$, and a moderate acceleration search in the Fourier domain (the zmax parameter was set to 50 in PRESTO analyses) to be sensitive to pulsars in binary systems. Searches for periodic signals in the data from this survey so far led to the discovery of one "ordinary" (i.e., non-millisecond) pulsar, PSR J2048+49, and two MSPs, PSRs J2055+3829 and J2205+6012. Details on the survey, the data analysis, and the discovered pulsars will be reported in Desvignes et al. (in prep.). In the present paper we report on the results from the timing of PSR J2055+3829, an MSP in an eclipsing BW system, and from the analysis of the radio eclipses of the pulsar. In Sect. 2 we describe the radio timing observations and the results from the analysis of the timing data. In Sect. 3 we present observations of eclipses of PSR J2055+3829 at $1.4 \mathrm{GHz}$, and analyses of the data taken around superior conjunction of the pulsar. In the following section (Sect. 4), we present comparisons of the mass function distributions for eclipsing and noneclipsing BWs, and for Galactic disk and globular cluster BWs. Finally, Sect. 5 summarizes our findings.

\section{Observations and data analysis}

\subsection{Radio observations and timing analysis}

The discovery of PSR J2055+3829 was described in detail by Octau (2017). In summary, the analysis of an 18-min observation conducted as part of the SPAN512 survey on 5 December 2013 (MJD 56631) at $1.4 \mathrm{GHz}$ with PRESTO revealed a candidate pulsar with a period of $2.08 \mathrm{~ms}$ and a DM of $91.9 \mathrm{pc} \mathrm{cm}^{-3}$. Confirmation observations of the same sky direction $(\mathrm{RA}=20: 55: 04$, $\operatorname{Dec}=+38: 37: 23)$ were carried out with the NRT on 19 October 2015 (MJD 57314) for $14 \mathrm{~min}$ and on 20 October 2015 (MJD 57315) for $67 \mathrm{~min}$; both of these observations resulted in firm detections of the pulsar and found a statistical significance well above $10 \sigma$. After the first few days of timing of the MSP, it became clear that it is in a compact, low-eccentricity orbit around a very low mass companion, and has an orbital period of $3.1 \mathrm{~h}$.

At that stage, our initial timing solution for PSR J2055+3829 enabled us to time the pulsar accurately over the typical duration of NRT pulsar observations of $1 \mathrm{~h}$. We thus commenced regular timing observations of the new MSP. The bulk of the observations were made at $1.4 \mathrm{GHz}$ (with an exact central frequency of $1484 \mathrm{MHz}$ ), and a few observations at higher frequencies of $2154 \mathrm{MHz}$ or $2539 \mathrm{MHz}$ were also conducted. The latter observations resulted in much weaker detections of the

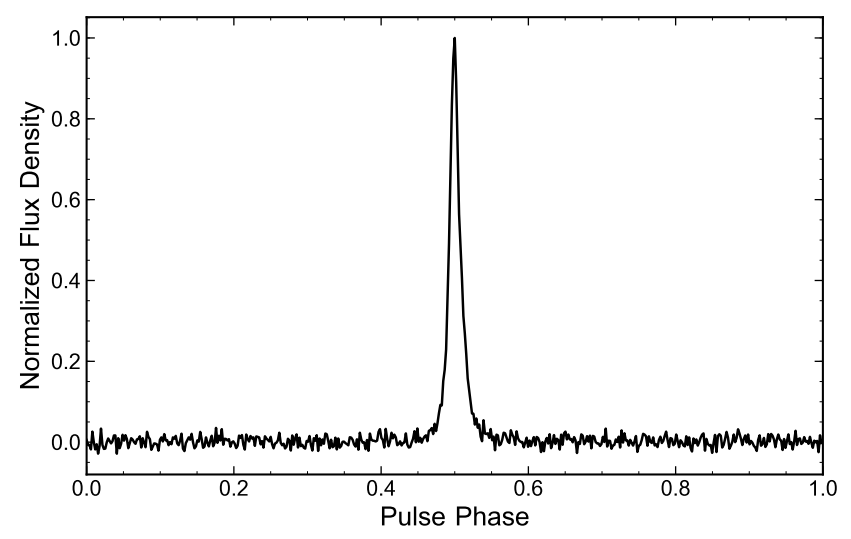

Fig. 1. Pulse profile for PSR J2055+3829 at $1.4 \mathrm{GHz}$, formed by integrating $48.8 \mathrm{~h}$ of coherently dedispersed observations with the Nançay Radio Telescope and the NUPPI backend. Profiles recorded at orbital phases between 0.1 and 0.4 were excluded from the summation to avoid contamination of the integrated profile from dispersive delays caused by ionized material near the companion star. To improve readability we divided the number of phase bins by four to form a profile with 512 phase bins.

pulsar; hence, most of the observations presented in this work were done at $1.4 \mathrm{GHz}$. We used the NUPPI pulsar observation backend, a version of the Green Bank Ultimate Pulsar Processing Instrument ${ }^{1}$ designed for Nançay (see Cognard et al. 2013, for a description). In these timing observations, 128 channels of $4 \mathrm{MHz}$ each are coherently dedispersed in real time and the time series are folded online at the expected topocentric pulsar period. We used the PSRCHIVE software library (Hotan et al. 2004) to clean the data of radio frequency interference and used the SINGLEAxIS method of PSRCHIVE to calibrate the polarization information. High signal-to-noise (S/N) NUPPI observations of PSR J2055+3829 made in timing mode at $1.4 \mathrm{GHz}$ were combined to form an average pulse profile, shown in Fig. 1. As is discussed later, the pulsar exhibits eclipses around superior conjunction (defined as orbital phase 0.25 ) and extra delays in the radio pulses recorded during eclipse ingress and egress caused by ionized material near the companion star were measured. We therefore conservatively excluded data at orbital phases between 0.1 and 0.4 when forming the average pulse profile. A total of $48.8 \mathrm{~h}$ of coherently dedispersed data were summed in the process. As can be seen from the figure, the pulse profile of the pulsar at $1.4 \mathrm{GHz}$ consists of a single, slightly asymmetric sharp peak, and there is no evidence for a secondary emission component. For the observations summed in the process we measured an average radio flux density at $1.4 \mathrm{GHz}$ of $S_{1400}=$ $0.10 \pm 0.04 \mathrm{mJy}$, where the uncertainty reflects the standard deviation of the individual flux density measurements. We note that since a large number of individual flux density measurements were used in the calculation, the average flux density quoted above is unlikely to be biased by scintillation effects.

A high S/N reference profile for PSR J2055+3829 at $1.4 \mathrm{GHz}$ was generated by smoothing the integrated pulse profile shown in Fig. 1, and times of arrival (TOAs) were generated by crosscorrelating the reference profile with the individual profiles. This cross-correlation was performed using the Fourier domain with Markov chain Monte Carlo algorithm implemented in the pat routine of PSRCHIVE, which properly estimates TOA uncertainties in the low $\mathrm{S} / \mathrm{N}$ regime. For each observation, we generated one TOA per $10 \mathrm{~min}$ and per $128 \mathrm{MHz}$ of bandwidth, so that

1 https://safe.nrao.edu/wiki/bin/view/CICADA/NGNPP 
Table 1. Parameters for PSR J2055+3829 derived from the analysis of the NRT timing data using TEMPO2.

\begin{tabular}{|c|c|c|}
\hline \multicolumn{3}{|c|}{ Data reduction parameters } \\
\hline Analysis type & Single template & 2D template \\
\hline Span of timing data (MJD) & $57333-58421$ & $57333-58421$ \\
\hline Number of TOAs & 1723 & 835 \\
\hline Weighted RMS residual $(\mu \mathrm{s})$ & 2.284 & 2.516 \\
\hline EFAC & 1.074 & 1.726 \\
\hline EQUAD $(\mu s)$ & 0.000 & 0.000 \\
\hline Reduced $\chi^{2}$ & 1.000 & 1.000 \\
\hline \multicolumn{3}{|c|}{ Astrometric and spin parameters } \\
\hline Right ascension, $\alpha(\mathrm{J} 2000)$ & $20: 55: 10.306550(4)$ & $20: 55: 10.306556(4)$ \\
\hline Declination, $\delta(\mathrm{J} 2000)$ & $+38: 29: 30.90571(6)$ & $+38: 29: 30.90571(6)$ \\
\hline Proper motion in $\alpha, \mu_{\alpha} \cos (\delta)\left(\right.$ mas $\left.\mathrm{yr}^{-1}\right)$ & $5.92(3)$ & $5.87(5)$ \\
\hline Proper motion in $\delta, \mu_{\delta}\left(\operatorname{mas}_{\mathrm{yr}^{-1}}\right)$ & $0.79(7)$ & $0.9(1)$ \\
\hline Spin frequency, $v(\mathrm{~Hz})$ & $478.631427595910(5)$ & $478.63142759590(2)$ \\
\hline Spin frequency derivative, $\dot{v}\left(10^{-16} \mathrm{~Hz} \mathrm{~s}^{-1}\right)$ & $-2.290(1)$ & $-2.289(2)$ \\
\hline Dispersion measure, DM $\left(\mathrm{pc} \mathrm{cm}^{-3}\right)$ & $91.8295(2)$ & $91.8295(7)$ \\
\hline Dispersion measure derivative, DM1 $\left(\mathrm{pc} \mathrm{cm}^{-3} \mathrm{yr}^{-1}\right)$ & $-0.0047(2)$ & $-0.0049(7)$ \\
\hline Reference epoch (MJD) & 57900 & 57900 \\
\hline \multicolumn{3}{|c|}{ Binary parameters } \\
\hline Orbital period, $P_{b}$ (days) & $0.12959037294(1)$ & $0.12959037293(2)$ \\
\hline Projected semimajor axis of the pulsar orbit, $x$ (lt-s) & $0.0452618(2)$ & $0.0452616(3)$ \\
\hline Epoch of ascending node, $T_{\text {asc }}$ & $57900.06984171(6)$ & $57900.06984175(9)$ \\
\hline$\eta \equiv e \sin \omega\left(10^{-5}\right)$ & $0.9(6)$ & $0.7(10)$ \\
\hline$\kappa \equiv e \cos \omega\left(10^{-5}\right)$ & $0.5(4)$ & $1.3(6)$ \\
\hline Orbital period derivative, $\dot{P}_{b}\left(10^{-12}\right)$ & $-2.00(9)$ & $-2.0(1)$ \\
\hline \multicolumn{3}{|c|}{ Derived parameters } \\
\hline Orbital eccentricity, $e\left(10^{-5}\right)$ & $1.0(6)$ & $1.4(7)$ \\
\hline Mass function, $f\left(10^{-6} M_{\odot}\right)$ & $5.92832(7)$ & $5.9283(1)$ \\
\hline Minimum companion mass, $m_{\mathrm{c}, \min }\left(M_{\odot}\right)$ & 0.02290 & 0.02290 \\
\hline Total proper motion, $\mu_{\mathrm{T}}\left({\left.\operatorname{mas~} \mathrm{yr}^{-1}\right)}^{-1}\right.$ & $5.97(4)$ & $5.93(6)$ \\
\hline Galactic longitude, $l\left(^{\circ}\right)$ & \multicolumn{2}{|c|}{80.615} \\
\hline Galactic latitude, $b\left(^{\circ}\right)$ & \multicolumn{2}{|c|}{-4.259} \\
\hline DM-derived distance, $d(\mathrm{kpc})$ & \multicolumn{2}{|c|}{$4.6(9)$} \\
\hline Transverse velocity, $v_{\mathrm{T}}\left(\mathrm{km} \mathrm{s}^{-1}\right)$ & \multicolumn{2}{|c|}{$13(3)$} \\
\hline Spin period, $P(\mathrm{~ms})$ & \multicolumn{2}{|c|}{$2.08929030219107(2)$} \\
\hline Spin period derivative, $\dot{P}\left(10^{-22} \mathrm{~s} \mathrm{~s}^{-1}\right)$ & \multicolumn{2}{|c|}{$9.996(5)$} \\
\hline Intrinsic spin period derivative, $\dot{P}_{\text {int }}\left(10^{-22} \mathrm{~s} \mathrm{~s}^{-1}\right)$ & \multicolumn{2}{|c|}{$8(2)$} \\
\hline Spin-down power, $\dot{E}\left(10^{33} \mathrm{erg} \mathrm{s}^{-1}\right)$ & \multicolumn{2}{|c|}{$3.6(7)$} \\
\hline Surface magnetic field intensity, $B_{\mathrm{s}}\left(10^{7} \mathrm{G}\right)$ & \multicolumn{2}{|c|}{$4.2(4)$} \\
\hline Magnetic field intensity at light cylinder, $B_{\mathrm{LC}}\left(10^{4} \mathrm{G}\right)$ & \multicolumn{2}{|c|}{$4.3(4)$} \\
\hline
\end{tabular}

Notes. Numbers in parentheses are the nominal $1 \sigma$ statistical uncertainties in the last digits quoted. The reference epoch of the pulsar's spin and astrometric and DM parameters is MJD 57900, and was chosen to be close to the center of the timing dataset. Epochs are given in TCB, and the DE436 planetary ephemeris was used. The DM-derived distance was estimated using the YMW16 model of free electron density (Yao et al. 2017). We assumed a pulsar mass of $1.4 M_{\odot}$ for the calculation of minimal companion masses and a moment of inertia $I$ of $10^{45} \mathrm{~g} \mathrm{~cm}^{2}$ for the calculation of $\dot{E}, B_{\mathrm{s}}$, and $B_{\mathrm{LC}}$.

each TOA covers less than $10 \%$ of an orbit; we split the total frequency bandwidth into several $128 \mathrm{MHz}$ channels to track potential time variations of the DM. We analyzed the TOA data using the TEMPO 2 pulsar timing package (Hobbs et al. 2006). The measured topocentric TOAs were converted to Barycentric Coordinate Time (TCB) using the DE436 solar system ephemeris $^{2}$, and accounting for the known clock corrections for the NUPPI backend. For the timing analysis, we again discarded TOAs corresponding to orbital phases between 0.1 and 0.4 .

\footnotetext{
2 https://naif.jpl.nasa.gov/pub/naif/JUNO/kernels/spk/ de436s.bsp.lbl
}

The best-fit timing solution, which minimizes the differences between measured TOAs and those predicted by TEMPO2 (the so-called timing residuals), is presented in Table 1 (see results in the "Single template" column), and the timing residuals as a function of time are shown in Fig. 2. We used the ELL1 binary timing model (Lange et al. 2001), which is well-suited for the fitting of orbital parameters in this configuration, because PSR J2055+3829 is in a low-eccentricity orbit. In addition to the binary parameters, we fit for the pulsar's sky position, proper motion, spin frequency and its first time derivative, and the DM and its first time derivative. To account for potential biases in the determination of the TOA uncertainties, we used the EFAC and EQUAD factors of TEMPO2. The EQUAD parameter was 

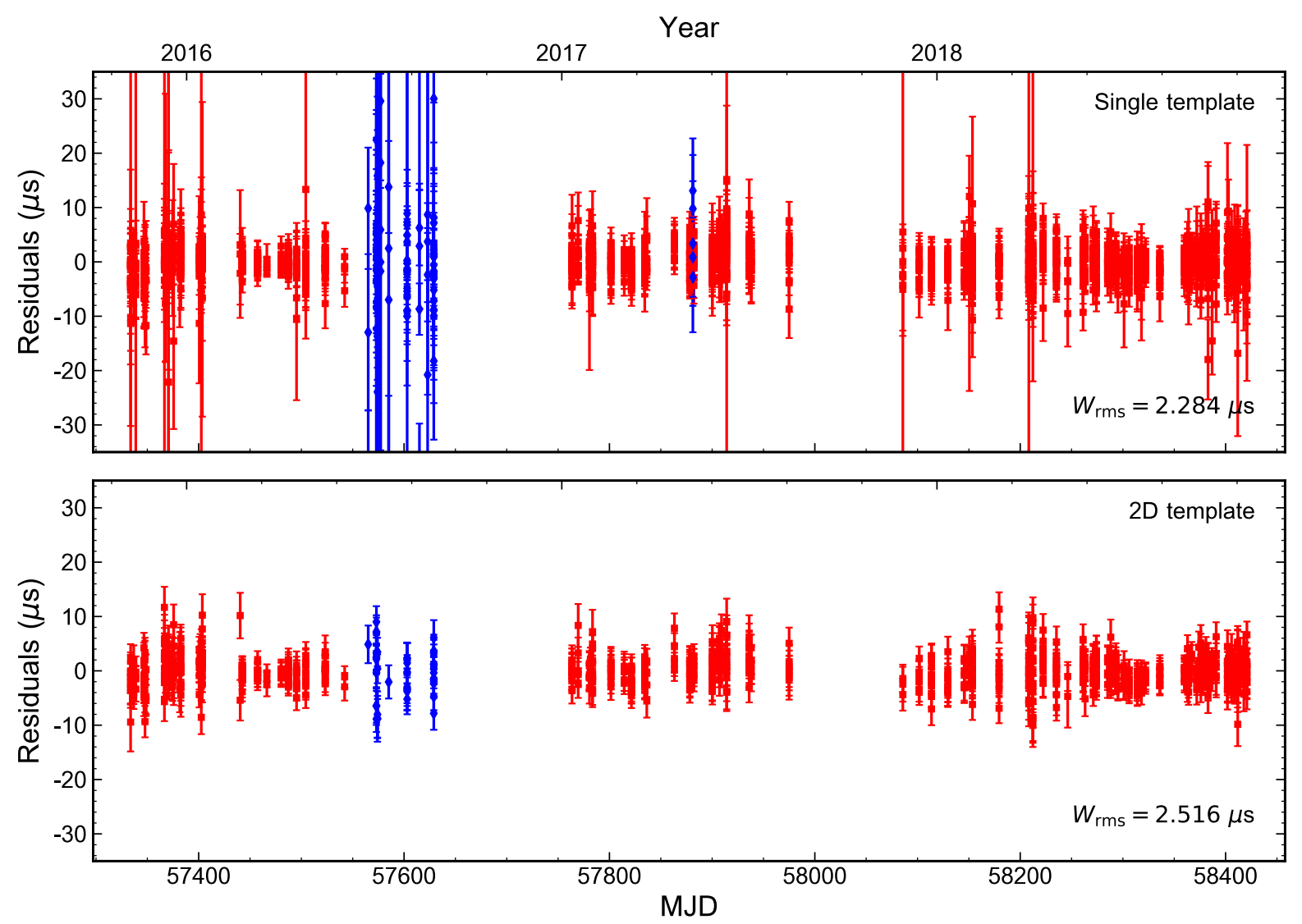

Fig. 2. Post-fit timing residuals as a function of time for the best-fit timing solutions presented in Table 1, considering non-eclipsing binary phases only. Residuals shown in the upper panel are based on TOAs extracted by using a single template profile representative of the full 512-MHz bandwidth at $1.4 \mathrm{GHz}$, while those in the lower panel are based on TOAs obtained by fitting a template profile with the full frequency resolution to the data. Details on the TOA integration times, frequency bandwidths, and extraction procedures are given in Sect. 2 for the upper panel, and in Sect. 3 for the lower panel. Residuals shown as red squares (resp., blue diamonds) correspond to observations made at $1.4 \mathrm{GHz}$ (resp., 2.1 and $2.5 \mathrm{GHz}$ ). In the upper panel (respectively, the lower panel), TOA uncertainties were multiplied by a correction factor (EFAC, see Sect. 2.1), of 1.074 (resp., 1.726).

found to be negligible; we thus set it to 0 in the final fit. On the other hand, an EFAC very close to 1 was found, indicating realistic TOA uncertainties. As can be seen from Table 1, the best-fit Laplace-Lagrange parameters $\eta$ and $\kappa$ are only marginally significant. A fit with $\eta=\kappa=0$ (i.e., a circular orbit) leads to slightly higher $\chi^{2}$ and rms residual values, and best-fit parameters consistent with those listed in Table 1.

The DM of the pulsar is observed to vary, and we find that a simple linear model for the DM enables us to model the multifrequency TOAs adequately. The best-fit DM function from the timing analysis is plotted as a function of time in Fig. 3. Also shown in the figure are the results of a fit of constant DM values to 50-day segments of the TOAs. The constant DM values are consistent with those predicted by the simple linear model.

Given the DM and position of the pulsar, the YMW16 electron-density model of Yao et al. (2017) predicts a distance $d$ of $4.6 \pm 0.9 \mathrm{kpc}$, assuming an uncertainty of $20 \%$. The NE2001 model (Cordes \& Lazio 2002) also predicts a relatively large distance of about $4.4 \mathrm{kpc}$. At the DM-derived distance $d \sim 4.6 \mathrm{kpc}$, the total transverse velocity of the pulsar derived from the measured proper motion is $\sim 13 \mathrm{~km} \mathrm{~s}^{-1}$. This low value is relatively common among other recycled pulsars (Hobbs et al. 2005). We note that the low transverse velocity of PSR J2055+3829 combined with the rate of change of the DM of $\sim 5 \times$ $10^{-3} \mathrm{pc} \mathrm{cm}^{-3} \mathrm{yr}^{-1}$, which is relatively high for an MSP (see, e.g., Jones et al. 2017), suggest that the pulsar could be undergoing

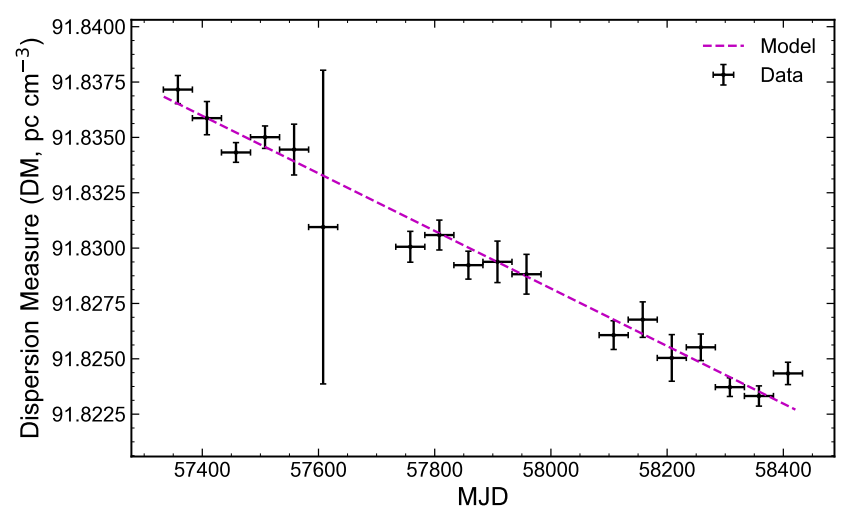

Fig. 3. Evolution of the DM of PSR J2055+3829 as a function of time. Data points shown in black were determined by fitting constant DM values to 50-day segments of NUPPI timing data. The NUPPI observations were split into four sub-bands of $128 \mathrm{MHz}$ each to allow for the fit in DM. The dashed line shows the best-fit DM model, as determined in the timing analysis (see Table 1, "Single template" column). The data point at MJD 57600 corresponds to a time interval in which only high frequency ( 2.1 and $2.5 \mathrm{GHz})$ observations were available, hence the large DM uncertainty.

radial motion through a region with a strong free electron density gradient. The modest transverse proper motion leads to a small Shklovskii correction (Shklovskii 1970) to the apparent 
spin period derivative, $\dot{P}$, calculated as

$\dot{P}_{\text {Shk }} \simeq 2.43 \times 10^{-21}\left(\frac{\mu_{\perp}}{\text { mas yr }^{-1}}\right)\left(\frac{d}{1 \mathrm{kpc}}\right)\left(\frac{P}{\mathrm{~s}}\right)$,

where $\mu_{\perp}$ is the total transverse proper motion of the pulsar. We accounted for this kinematic effect and for the correction caused by the difference in Galactic accelerations of the pulsar and the solar system, using the model for the rotation of the Galaxy from Carlberg \& Innanen (1987) and Kuijken \& Gilmore (1989), to derive the intrinsic spin period derivative $\dot{P}_{\text {int }}$ quoted in Table 1. The latter spin-down rate value was used to infer the pulsar's spin-down power $\dot{E}$, and magnetic field intensities at the stellar surface, $B_{\mathrm{s}}$, and at the light cylinder, $B_{\mathrm{LC}}$.

Using the measured projected semimajor axis of the pulsar orbit, $x$, and the orbital period, $P_{b}$, we calculated the mass function given in Table 1, as

$f\left(m_{\mathrm{p}}, m_{\mathrm{c}}\right)=\frac{\left(m_{\mathrm{c}} \sin i\right)^{3}}{\left(m_{\mathrm{p}}+m_{\mathrm{c}}\right)^{2}}=\frac{4 \pi}{T_{\odot}} \frac{x^{3}}{P_{b}^{2}}$,

where $m_{\mathrm{p}}$ is the pulsar mass, $m_{\mathrm{c}}$ is the companion mass, $i$ is the inclination of the orbit, and $T_{\odot}=G M_{\odot} / c^{3}=4.925490947 \mu \mathrm{s}$. Assuming a canonical mass of $1.4 M_{\odot}$ and an edge-on orbit $\left(i=90^{\circ}\right)$, we obtain a lower limit on the companion mass of $\sim 0.023 M_{\odot}$. Since the probability of observing a binary system with an inclination lower than $i_{0}$ for a random distribution of inclinations is given by $1-\cos \left(i_{0}\right)$, a $90 \%$ confidence upper limit on the companion mass can be determined by assuming an inclination of $26^{\circ}$. We find an upper limit on the companion mass of $\sim 0.053 M_{\odot}$. As can be noted from Table 1 , the analysis of the NRT timing dataset revealed a significant $\dot{P}_{b}$ term, indicative of orbital period variations. These variations, which are commonly observed in other BW systems such as PSRs B1957+20, J1731-1847, or J2051-0827 (see for instance Bates et al. 2011; Shaifullah et al. 2016, and references therein), the very low companion mass and the observation of eclipses around superior conjunction (see Sect. 3) firmly establish PSR J2055+3829 as a BW pulsar.

\subsection{Gamma-ray analysis}

The pulsar's spin-down power value $\dot{E} \sim 4 \times 10^{33} \mathrm{erg} \mathrm{s}^{-1}$ is above the empirical deathline for gamma-ray emission from MSPs (Guillemot et al. 2016), and is comparable to that of many gamma-ray-detected MSPs (see for example Abdo et al. 2013; Smith et al. 2019). PSR J2055+3829 is thus a candidate for a detection in gigaelectronvolt $(\mathrm{GeV})$ gamma rays with the Fermi-LAT (Atwood et al. 2009). No gamma-ray counterpart is found in the recently released Fermi-LAT 8-year Source Catalog (4FGL; see Fermi-LAT Collaboration 2019) within $2^{\circ}$ of PSR J2055+3829. However, since pulsation searches are more sensitive than searches for continuous gamma-ray emission (see Smith et al. 2019, for recent examples of gamma-ray pulsars not reported in LAT source catalogs), we searched the LAT data for gamma-ray pulsations from PSR J2055+3829. We analyzed $\sim 10.2$ years of Pass 8 SOURCE class Fermi-LAT events, with energies from $0.1-100 \mathrm{GeV}$, found within $3^{\circ}$ of the pulsar's sky position, and with zenith angles smaller than $105^{\circ}$. Pulse phases were calculated using the fermi plugin of TEMPO2 (Ray et al. 2011) and the timing solution described above. The sensitivity of our gamma-ray pulsation searches was enhanced using the photon weighting scheme described in Bruel (2019), and we followed the same strategy as described in Smith et al.
(2019) for searching the value of the $\mu_{\mathrm{w}}$ weighting parameter that maximizes the significance of gamma-ray pulsations; that is, we tested three values of $\mu_{\mathrm{w}}$, i.e., $3.2,3.6$, and 4.0, on the full LAT dataset available (MJD 54682 until MJD 58421) and on the validity interval of the timing solution (MJD 57333 to MJD 58421). None of the six search trials resulted in a pulsation significance above $2 \sigma$. With its Galactic latitude of $-4.259^{\circ}$, PSR J2055+3829 lies close to the Galactic plane and is also located near the gamma-ray-bright Cygnus region. Because of the relatively large point-spread function of the LAT at low energies, the selected dataset was likely contaminated by a large number of background gamma-ray photons. We repeated the analysis described above using a smaller event selection radius of $1^{\circ}$ and obtained consistent results.

The non-detection of gamma-ray pulsations may result from the fact that the current timing solution for PSR J2055+3829 is not able to maintain phase-connection over the entire FermiLAT dataset, thus preventing us from detecting faint pulsations. Unfavorable beaming geometry could also explain the absence of significant gamma-ray pulsations. Guillemot \& Tauris (2014) for instance found marginal evidence for different viewing angle (the angle between the spin axis and the line of sight) distributions between gamma-ray-detected and undetected energetic and nearby MSPs, and postulated that the undetected MSPs are seen under small viewing angles. However, a more likely explanation for the non-detection resides in the large distance of the pulsar compared to those of known gamma-ray MSPs. The DM distance estimated with the YMW16 model of $\sim 4.6 \mathrm{kpc}$, if close to the actual value, is indeed significantly larger than the typical distance of gamma-ray MSPs of $\sim 1 \mathrm{kpc}$ (see, e.g., Abdo et al. 2013). The lack of a counterpart in 4FGL within $2^{\circ}$ of PSR J2055+3829 means that the integrated energy flux above $0.1 \mathrm{GeV}$ for this pulsar is less than $4 \times 10^{-12} \mathrm{erg} \mathrm{cm}^{-2} \mathrm{~s}^{-1}$; this value was obtained with the same method as the three-year sensitivity map in Fig. 16 of Abdo et al. (2013), but used the eight-year 4FGL model extrapolated to the $\sim 10.2$ years of data used in this work. This energy flux limit is smaller than the fluxes of LAT-detected MSPs at Galactic latitudes within $5^{\circ}$ of that of PSR J2055+3829 (see 4FGL). Besides, assuming that the pulsar has an efficiency of conversion of spin-down power $\dot{E}$ into gamma-ray luminosity $L_{\gamma}$ of $100 \%$ leads to an expected gamma-ray energy flux $h=\dot{E} /\left(4 \pi d^{2}\right)$ of $1.4 \times 10^{-12} \mathrm{erg} \mathrm{cm}^{-2} \mathrm{~s}^{-1}$ at the distance of $4.6 \mathrm{kpc}$, smaller than the energy flux limit quoted above. The detection of pulsed high-energy emission from PSR J2055+3829 would thus require significantly more gamma-ray data or a favorable (i.e., sharp) gamma-ray pulse profile allowing detection in spite of faint emission (see Hou et al. 2014, for discussion).

\section{Eclipse properties}

As mentioned above, our $1.4 \mathrm{GHz}$ observations of PSR J2055+3829 revealed the presence of eclipses of the radio signal of the pulsar around superior conjunction. A selection of high $\mathrm{S} / \mathrm{N}$ detections of the pulsar containing complete or partial eclipse traverses is shown in Fig. 4. It is apparent from this figure that the pulsar emission is completely obscured at $1.4 \mathrm{GHz}$ for a few minutes around orbital phase 0.25 . As is commonly observed in other BW systems displaying eclipses (e.g., Bates et al. 2011; Bhattacharyya et al. 2013; Polzin et al. 2018), we see asymmetric phase modulations in the pulsar signal at ingress and egress. The slightly longer egress phases suggest that the orbital motion of the companion causes its wind to be swept back (Fruchter et al. 1990; Stappers et al. 2001). 

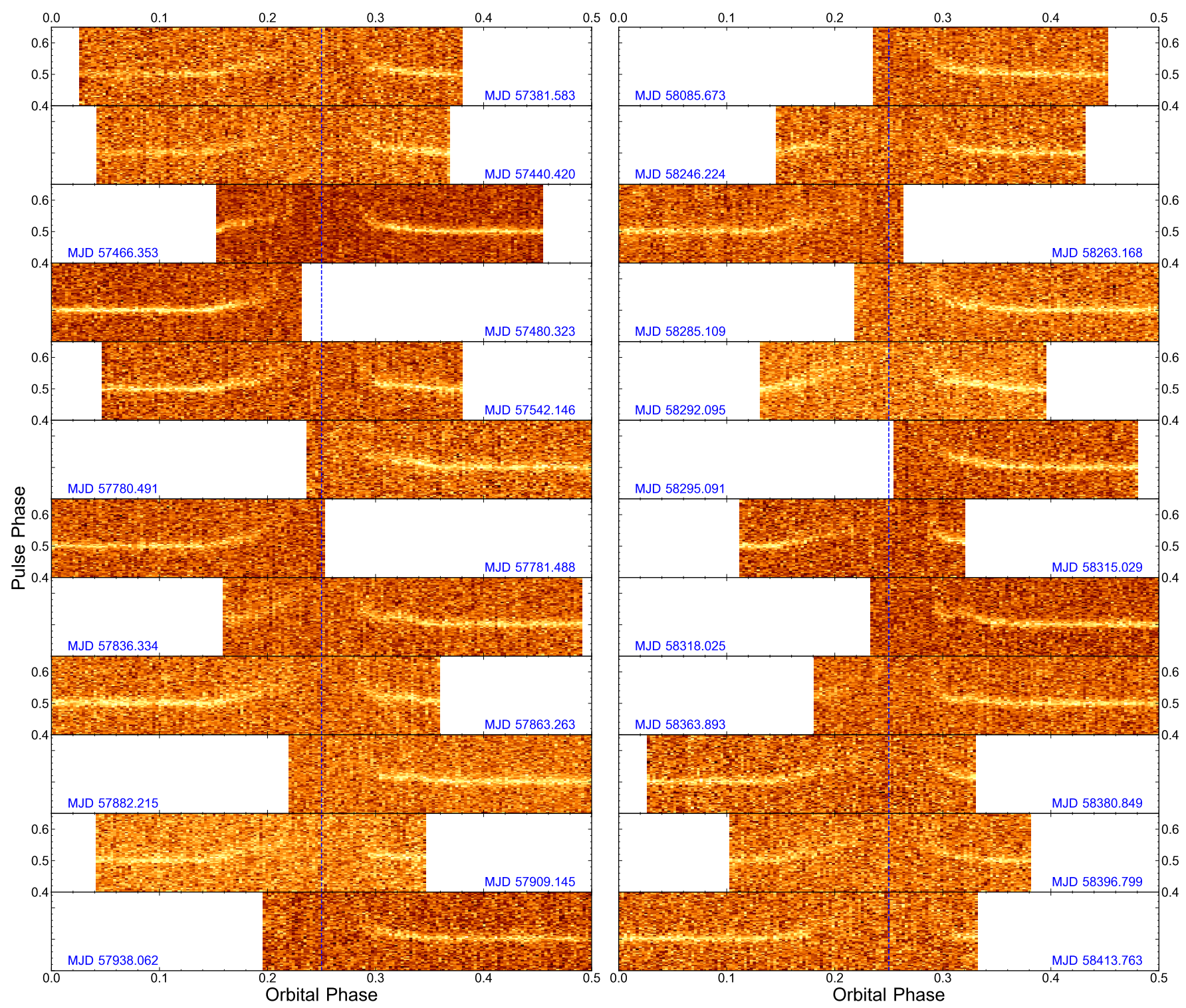

Fig. 4. Flux density as a function of orbital and pulse phase, for several observations of PSR J2055+3829 at $1.4 \mathrm{GHz}$ with the NUPPI backend. Observation start epochs are given in each panel. To improve readability, the number of phase bins was reduced by a factor of eight, and the time-resolution was decreased to form one profile per approximately $30 \mathrm{~s}$. The dashed blue lines indicate the phase of superior conjunction (orbital phase 0.25).

The observations shown in Fig. 4 also indicate variations in the duration of the individual eclipses, as well as short-duration absorption events at egress (e.g., at MJD 58318) indicative of clumpiness in the outflow from the companion. More sensitive observations or observations at lower radio frequencies would be useful for characterizing the eclipse-to-eclipse variability further.

For the timing analysis presented in Sect. 2, we used a TOA dataset comprising one TOA per $10 \mathrm{~min}$ and per $128 \mathrm{MHz}$ with a total bandwidth of $512 \mathrm{MHz}$. With such a time- and frequencyresolution we could track long-term DM variations while keeping integration times short compared to the orbital period of the pulsar. Nevertheless, to investigate short-term variations of the DM around superior conjunction with increased sensitivity and time resolution, we used the wide-band template matching technique implemented in the PulsePortraiture software library ${ }^{3}$

\footnotetext{
3 https://github.com/pennucci/PulsePortraiture
}

(Pennucci et al. 2014) to extract one TOA per 5 min of observation for the entire frequency bandwidth. The two-dimensional template profile was constructed by summing 40 observations of PSR J2055+3829 well outside of its eclipses, keeping the full frequency resolution available. The results of the timing analysis with this new TOA dataset are given in Table 1 (see " $2 \mathrm{D}$ template" column), and the corresponding timing residuals are plotted in Fig. 2. With the notable exception of the EFAC parameter of $\sim 1.73$, indicative of TOA uncertainties that were likely underestimated, the parameters from this new timing analysis are consistent with those obtained with the standard TOA extraction technique presented in Sect. 2.

In addition to extracting the TOAs, we used the wide-band template matching technique to measure the DM of the pulsar in each five-min data sample. The timing residuals and the DM values from this analysis around the eclipse phases are shown in Fig. 5. Dispersion measure values plotted in this figure were corrected for the long-term time variation measured from the 


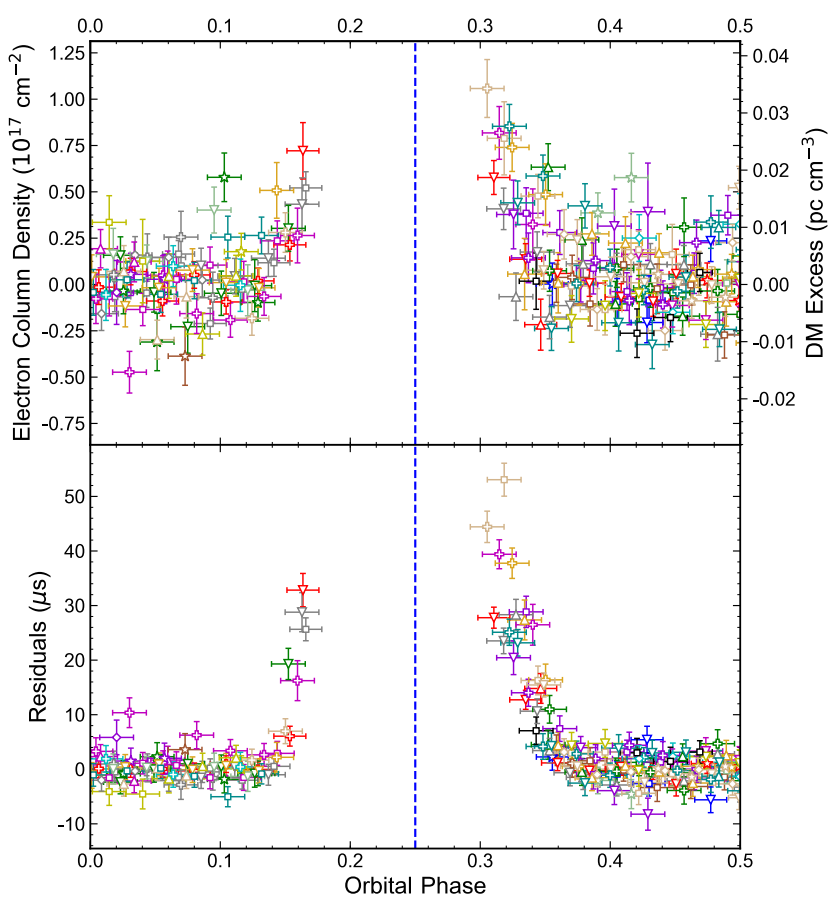

Fig. 5. Timing residuals and excess electron column density as a function of orbital phase, around superior conjunction. All data points in this figure correspond to $1.4 \mathrm{GHz}$ observations; S/Ns larger than 19 are close to the median value. Residuals and electron column densities from a given observation are shown with the same combination of symbols and colors. The phase of superior conjunction is shown as a dashed blue line.

timing analysis and corresponding to the linear model plotted in Fig. 3. The DM offsets are converted to excess electron column densities, assuming that the material causing the delays is a homogeneous plasma. The eclipses are centered at orbital phase $\sim 0.24$ and have a duration of about 19 min or $\sim 10 \%$ of the orbital period, where the center phase and duration quoted simply correspond to the middle and separation of the last TOA before and first TOA after the eclipse. The asymmetry of the ingress and egress phases mentioned earlier is apparent in the timing residuals. To a good approximation, the radius of the companion's Roche lobe can be estimated to be (Eggleton 1983)

$R_{\mathrm{L}}=\frac{0.49 a q^{2 / 3}}{0.6 q^{2 / 3}+\ln \left(1+q^{1 / 3}\right)}$,

where $R_{\mathrm{L}}$ the radius of the Roche lobe, $q=m_{\mathrm{c}} / m_{\mathrm{p}}$ is the companion and pulsar mass ratio, and $a$ is the separation between the companion star and the pulsar. Assuming $m_{\mathrm{p}}=1.4 M_{\odot}$ and the minimum companion mass $m_{\mathrm{c}, \min }=0.02290 M_{\odot}$, we find $a \sim 1.2 R_{\odot}$, and $R_{\mathrm{L}} \sim 0.14 R_{\odot}$. The eclipse duration translates to an opaque fraction of the companion's orbit of $\sim 0.75 R_{\odot}$, which is larger than the Roche lobe radius of the companion. This indicates that the eclipsing material is not gravitationally bound to the companion. At $1.4 \mathrm{GHz}$, the maximum added electron density near superior conjunction is found to be $N_{\mathrm{e}, \max } \sim 10^{17} \mathrm{~cm}^{-2}$.

Table 4 of Bates et al. (2011) lists a number of useful eclipse properties for the extensively studied eclipsing BW pulsars in the Galactic disk J1731-1847, B1957+20, and J2051-0827. Similar information on the eclipsing BWs PSR J1544+4937 and $\mathrm{J} 1810+1744$ can be found in Bhattacharyya et al. (2013) and Polzin et al. (2018), respectively, although the latter articles did not report any orbital period derivative measurement. Comparing the values of the eclipse radius, Roche lobe radius, $P_{b}$,
$\left|\dot{P}_{b}\right| / P_{b}, \dot{E} / a^{2}$, and $N_{\mathrm{e}, \max }$ for PSR J2055+3829 with those of other eclipsing BW pulsars, we find that the new MSP is similar to the previously reported MSP in most respects. The eclipse radius, Roche lobe radius, and maximum excess electron density of PSR J2055+3829 are comparable to those of other eclipsing BWs. For the new MSP we find $\dot{E} / a^{2} \sim 4.6 \times 10^{32} \mathrm{erg} \mathrm{s}^{-1} \mathrm{lt}-$ $\mathrm{s}^{-2}$. Although this value for the $\dot{E} / a^{2}$ parameter is the lowest among those of the pulsars in this category, it is very close to the value for PSR J2051-0827 of $4.8 \times 10^{32} \mathrm{erg} \mathrm{s}^{-1} 1 \mathrm{t}-\mathrm{s}^{-2}$, and is still several orders of magnitude higher than the median $\dot{E} / a^{2}$ value for other pulsars in binary systems. As was noted by Bates et al. (2011), this strong energy flux at the distance of the companion could explain why ablation occurs in BW systems and not in other binaries. Finally, we find that our value of $\left|\dot{P}_{b}\right| / P_{b}$ is an order of magnitude lower than those of PSRs J1731-1847, B1957+20, and J2051-0827. Although statistically significant, the $\dot{P}_{b}$ value given in Table 1 should be taken with a grain of salt since the $\dot{P}_{b}$ values of BWs are known to vary rapidly (see, e.g., Fig. 5 of Shaifullah et al. 2016, for the case of PSR J2051-0827). The many similarities PSR J2055+3829 shares with other known eclipsing BWs suggest that they were formed by a common process. Observations of this new MSP thus provide new insight into the origin of the population of BWs and into eclipse mechanisms in these objects.

\section{Mass functions of eclipsing and non-eclipsing black widows}

Freire (2005) compared the properties of eclipsing binary pulsars in the Galactic disk and in globular clusters (GCs) and found evidence for eclipsing BWs having higher mass functions than non-eclipsing ones. This can be understood as follows: eclipsing systems are thought to be seen under higher inclinations than non-eclipsing systems. Since the mass function is proportional to $(\sin i)^{3}$ (see Eq. (2)) and assuming that the pulsar and companion masses in these systems are comparable to those in eclipsing systems, non-eclipsing BWs are thus expected to have lower mass functions.

Motivated by the fact that the number of known BW pulsars in the Galactic disk and in GCs has increased substantially since the Freire (2005) study was published, we revisited the mass function distributions of eclipsing and non-eclipsing BWs. We selected Galactic disk ${ }^{4}$ and $\mathrm{GC}^{5}$ BW MSPs (here defined as MSPs orbiting objects with masses $m_{\mathrm{c}}<0.06 M_{\odot}$, and not known to be planets), with published information regarding the presence of radio eclipses or lack thereof. Table 2 lists the selected objects, and some of their main orbital properties. The orbital parameters quoted in Table 2 were taken from the Australian Telescope National Facility (ATNF) pulsar database ${ }^{6}$ (Manchester et al. 2005) when available, otherwise they were taken from the public lists of Galactic disk and GC MSPs. Cumulative histograms of the mass functions of eclipsing and noneclipsing BWs in the Galactic disk, in GCs, and of the total population of known BWs are shown in Fig. 6. One-dimensional Kolmogorov-Smirnov (KS) tests (Press et al. 1992) indicate that the probability that eclipsing and non-eclipsing objects originate from the same parent distribution is about $1.5 \%$ in the case of Galactic disk MSPs, only $\sim 0.08 \%$ for GC BWs, and $\sim 0.007 \%$

\footnotetext{
4 A list of Galactic disk MSPs is available at http://astro.phys. wvu . edu/GalacticMSPs/GalacticMSPs . txt

5 See http://www. naic. edu/ pfreire/GCpsr.html for a list of known GC pulsars and their main properties.

6 http://WwW.atnf.csiro.au/people/pulsar/psrcat/
} 
Table 2. Orbital properties of Galactic disk and GC BW pulsars.

\begin{tabular}{|c|c|c|c|c|}
\hline Pulsar & $P_{b}$ (days) & $x(1 \mathrm{t}-\mathrm{s})$ & $f\left(10^{-6} M_{\odot}\right)$ & Eclipses? \\
\hline \multicolumn{5}{|c|}{ Galactic disk black widow pulsars } \\
\hline $\mathrm{J} 0023+0923$ & 0.139 & 0.035 & 2.36 & - \\
\hline $\mathrm{J} 0251+2606$ & 0.204 & 0.066 & 7.42 & $\mathrm{Y}$ \\
\hline J0610-2100 & 0.286 & 0.073 & 5.21 & - \\
\hline J0636+5129 & 0.067 & 0.009 & 0.18 & - \\
\hline J0952-0607 & 0.267 & 0.063 & 3.69 & - \\
\hline J1124-3653 & 0.227 & 0.080 & 10.67 & $\mathrm{Y}$ \\
\hline $\mathrm{J} 1301+0833$ & 0.272 & 0.078 & 6.89 & Y \\
\hline J1311-3430 & 0.065 & 0.011 & 0.30 & $\mathrm{Y}$ \\
\hline J1446-4701 & 0.278 & 0.064 & 3.65 & - \\
\hline $\mathrm{J} 1513-2550$ & 0.179 & 0.041 & 2.31 & $\mathrm{Y}$ \\
\hline $\mathrm{J} 1544+4937$ & 0.121 & 0.033 & 2.61 & $\mathrm{Y}$ \\
\hline J1731-1847 & 0.311 & 0.120 & 19.24 & $\mathrm{Y}$ \\
\hline $\mathrm{J} 1745+1017$ & 0.730 & 0.088 & 1.38 & - \\
\hline $\mathrm{J} 1805+0615$ & 0.338 & 0.088 & 6.42 & - \\
\hline $\mathrm{J} 1810+1744$ & 0.148 & 0.095 & 42.03 & $\mathrm{Y}$ \\
\hline J1946-5403 & 0.130 & 0.043 & 5.23 & - \\
\hline B1957+20 & 0.382 & 0.089 & 5.23 & $\mathrm{Y}$ \\
\hline J2017-1614 & 0.098 & 0.043 & 8.89 & $\mathrm{Y}$ \\
\hline J2051-0827 & 0.099 & 0.045 & 10.01 & $\mathrm{Y}$ \\
\hline $\mathrm{J} 2052+1219$ & 0.115 & 0.061 & 18.43 & $\mathrm{Y}$ \\
\hline $\mathrm{J} 2055+3829$ & 0.130 & 0.045 & 5.93 & $\mathrm{Y}$ \\
\hline $\mathrm{J} 2115+5448$ & 0.135 & 0.044 & 5.02 & $\mathrm{Y}$ \\
\hline $\mathrm{J} 2214+3000$ & 0.417 & 0.059 & 1.28 & - \\
\hline $\mathrm{J} 2241-5236$ & 0.146 & 0.026 & 0.87 & - \\
\hline J2256-1024 & 0.210 & 0.081 & 12.94 & Y \\
\hline \multicolumn{5}{|c|}{ Globular cluster black widow pulsars } \\
\hline J0024-7204I & 0.230 & 0.038 & 1.16 & - \\
\hline J0023-7203J & 0.121 & 0.040 & 4.86 & $\mathrm{Y}$ \\
\hline $\mathrm{J} 0024-7204 \mathrm{O}$ & 0.136 & 0.045 & 5.35 & Y \\
\hline J0024-7204P & 0.147 & 0.038 & 2.72 & - \\
\hline J0024-7204R & 0.066 & 0.034 & 9.31 & $\mathrm{Y}$ \\
\hline $\mathrm{J} 1518+0204 \mathrm{C}$ & 0.087 & 0.057 & 26.82 & $\mathrm{Y}$ \\
\hline $\mathrm{J} 1641+3627 \mathrm{E}$ & 0.117 & 0.037 & 3.97 & Y \\
\hline $\mathrm{J} 1701-3006 \mathrm{E}$ & 0.159 & 0.070 & 14.76 & $\mathrm{Y}$ \\
\hline $\mathrm{J} 1701-3006 \mathrm{~F}$ & 0.205 & 0.057 & 4.78 & - \\
\hline $\mathrm{J} 1748-2446 \mathrm{O}$ & 0.260 & 0.112 & 22.40 & $\mathrm{Y}$ \\
\hline J1807-2459A & 0.071 & 0.012 & 0.39 & - \\
\hline $\mathrm{J} 1824-2452 \mathrm{G}$ & 0.105 & 0.017 & 0.44 & - \\
\hline $\mathrm{J} 1824-2452 \mathrm{~J}$ & 0.097 & 0.025 & 1.77 & - \\
\hline $\mathrm{J} 1824-2452 \mathrm{~L}$ & 0.226 & 0.057 & 3.90 & - \\
\hline $\mathrm{J} 1836-2354 \mathrm{~A}$ & 0.203 & 0.046 & 2.61 & - \\
\hline $\mathrm{J} 1911+0102 \mathrm{~A}$ & 0.141 & 0.038 & 2.88 & - \\
\hline $\mathrm{J} 1953+1846 \mathrm{~A}$ & 0.177 & 0.078 & 16.44 & $\mathrm{Y}$ \\
\hline
\end{tabular}

Notes. For each pulsar (see Sect. 4 for details on the selection criteria) we list the orbital period $P_{b}$, the projected semimajor axis $x$, and the mass function $f$. The last column indicates whether the pulsar is known to exhibit radio eclipses near superior conjunction.

for the total population of BWs. Therefore, the mass function distributions of eclipsing and non-eclipsing BWs appear to be drawn from distinct distributions in all three cases; eclipsing objects generally have higher mass functions than others. Two caveats we must point out are that not all MSPs considered as non-eclipsing have been observed at multiple radio frequencies (and in particular at low frequencies) so that some of them may eventually be found to exhibit eclipses, and the fact that non-eclipsing pulsars seen under low orbital inclinations may have heavier companions than actual BW pulsars. Despite these important caveats, this study gives credence to the hypothesis that eclipsing BWs have higher inclination angles (and thus higher mass functions) than non-eclipsing BWs. Simulations of populations of BW systems seen under many inclination angles and using realistic companion and pulsar mass distributions are beyond the scope of this paper, but may provide an avenue for investigating the observed differences further.

In addition to comparing the mass function distributions of eclipsing and non-eclipsing BWs, we also compared the mass functions of Galactic disk and GC BW pulsars (i.e., considering eclipsing and non-eclipsing pulsars), finding no obvious differences between their distributions. A comparison of the distributions (plotted in Fig. 7) with the KS test gives a probability that they originate from a common parent distribution of about $50 \%$, that is, Galactic disk and GC BWs appear to have consistent mass function distributions. This indicates strong similarities between the last stages of the evolution of BWs in the Galactic disk and in GCs. This is the case despite the very different conditions and interactions in these environments and particularly the fact that MSPs in GCs evolved from X-ray binaries formed by exchange interactions, unlike Galactic disk MSP systems that evolve from primordial binaries (see, e.g., Freire 2013).

\section{Summary}

We report on the timing of an MSP in a BW system, PSR J2055+3829, originally discovered as part of the SPAN512 pulsar survey conducted at the Nançay Radio Telescope. A number of these BW pulsars are known to exhibit complex orbital instabilities (see, e.g., Shaifullah et al. 2016, and references therein) and are thus not stable enough for PTA applications, although we note that Bochenek et al. (2015) presented arguments in favor of their inclusion in PTAs. Continued timing observations of PSR $\mathrm{J} 2055+3829$, whose apparent rotation appears to be relatively stable so far, will enable us to evaluate its long-term stability. Additionally, the long-term monitoring of this new pulsar may eventually make it possible to detect gamma-ray pulsations, although the low expected energy flux at the distance of $4.6 \mathrm{kpc}$ makes the detection of PSR J2055+3829 challenging, as discussed in Sect. 2.2.

At $1.4 \mathrm{GHz}$, the pulsar is observed to be eclipsing for about $10 \%$ of the orbit. As is also seen in other similar systems, the eclipses of PSR J2055+3829 are asymmetric and variable. We also find that a number of its eclipse properties (e.g., the eclipse radius, the maximum excess column density near superior conjunction, or the $\dot{E} / a^{2}$ flux parameter) resemble those of previously studied BW systems. More generally, we find significant differences between the mass function distributions of eclipsing and non-eclipsing BWs in the Galactic disk, in GCs, and for the total population of known BWs. Eclipsing BWs tend to have higher mass functions than non-eclipsing BWs, possibly because they are seen under higher inclination angles. On the other hand, Galactic disk and GC BWs have consistent mass function distributions, indicating that similar late phases of the evolutionary processes are at play in these different environments.

As mentioned in Sect. 3, more sensitive observations of PSR J2055+3829 or observations at lower frequencies, where the eclipses and the dispersion of the radio signal by the outflow from the companion are more pronounced, would be particularly useful for further characterizing the nature of the eclipses and the interactions between the pulsar flux and the plasma released by the companion star. For instance, by observing the original BW pulsar B1957+20 with the $305 \mathrm{~m}$ Arecibo telescope at $0.3 \mathrm{GHz}$, Main et al. (2018) were able to detect extreme plasma 

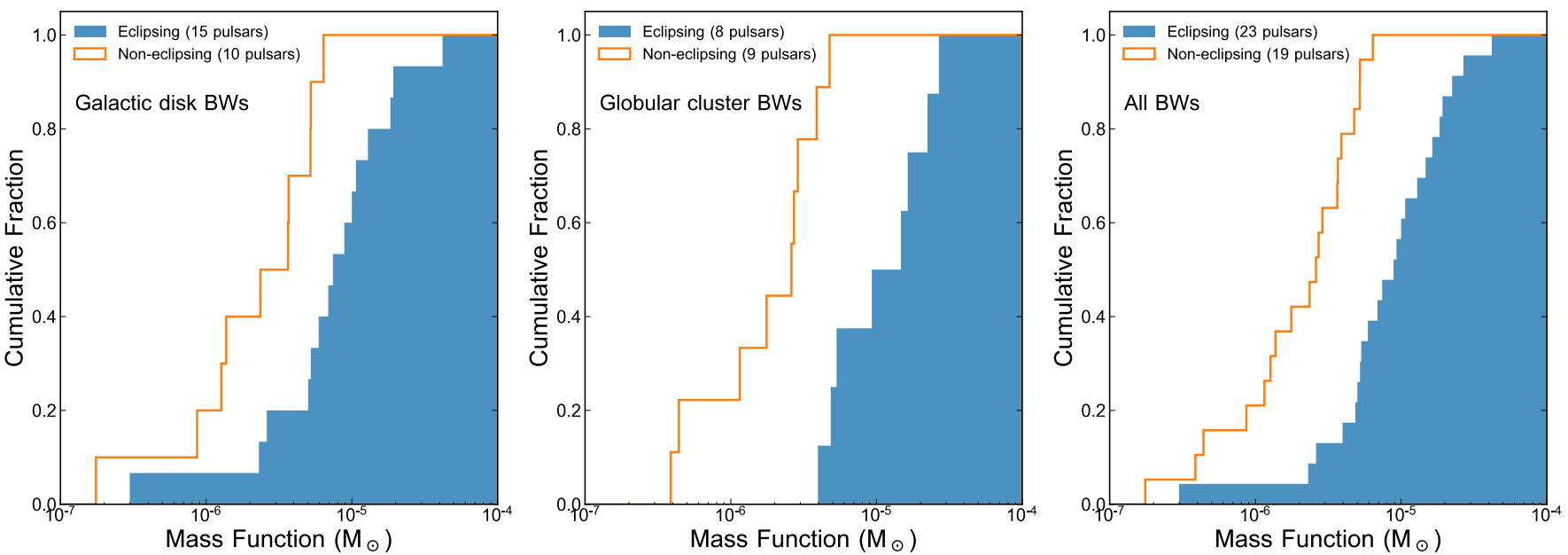

Fig. 6. Cumulative histograms of the mass functions (see Eq. (2)) of known eclipsing and non-eclipsing pulsars in the Galactic disk (left panel), in GCs (middle panel) and for the total population of BW pulsars (right panel). Pulsar names and mass functions for the various populations are given in Table 2.

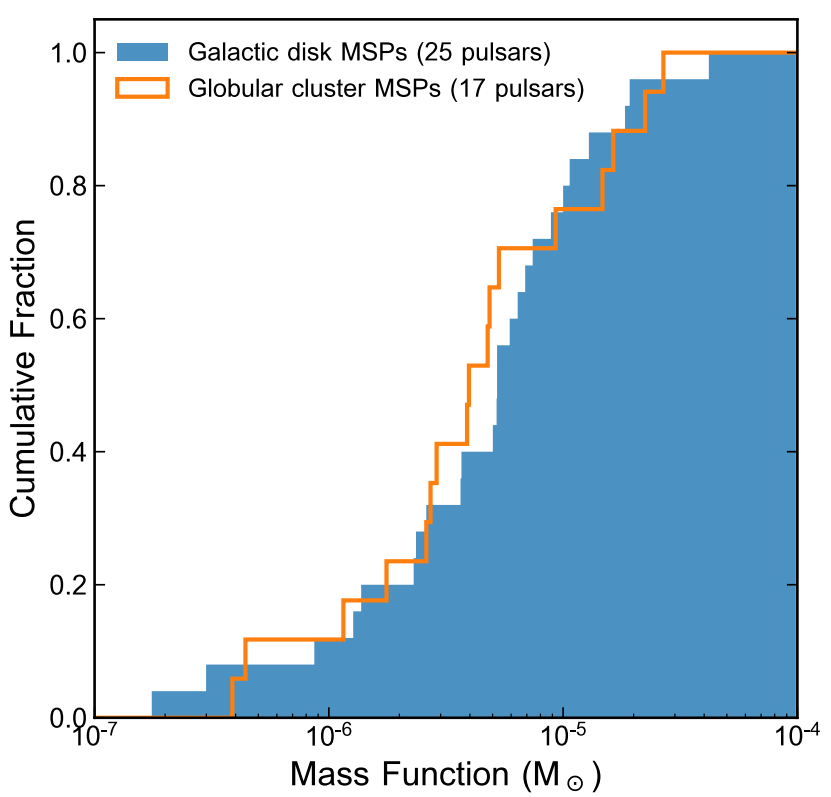

Fig. 7. Cumulative histograms of the mass functions of BW pulsars in the Galactic disk and in GCs.

lensing events near superior conjunction of the pulsar, making it possible to detect the pulsar during individual rotations and thus resolve its magnetosphere. Observations of plasma lensing events in B1957+20 were also used for probing the magnetic field at the interface between the pulsar and companion winds (Li et al. 2019). Another recent example is the low-frequency observations of the BW pulsar J1810+1744 with the Low Frequency Array (LOFAR) and Westerbork telescopes presented in Polzin et al. (2018), which enabled fine analyses of DM, flux density, and scattering time variations during eclipse traverses. Finally, we note that optical observations of BW systems can provide information on the heating of the companion and can be used to constrain the pulsar masses (e.g., Breton et al. 2013). X-ray observations of BW pulsars can also be used to study the emission arising from the intra-binary shock of the pulsar wind (see for instance Gentile et al. 2014), providing useful insight into the properties of this wind. No X-ray or optical observations of this pulsar have yet been conducted. New observa- tions of PSR J2055+3829 at complementary wavelengths and energies are therefore warranted.

Acknowledgements. The Nançay Radio Observatory is operated by the Paris Observatory, associated with the French Centre National de la Recherche Scientifique (CNRS). We acknowledge financial support from the "Programme National de Cosmologie and Galaxies" (PNCG), "Programme National Hautes Energies" (PNHE), and "Programme National Gravitation, Références, Astronomie, Métrologie" (PNGRAM) of CNRS/INSU, France. PCCF gratefully acknowledges financial support by the European Research Council for the ERC Starting grant BEACON and continued support from the Max Planck Society under contract No. 279702. We gratefully acknowledge support from the CNRS/IN2P3 Computing Center (CC-IN2P3Lyon/Villeurbanne) for providing the computing resources needed for the pulsar searches in the SPAN512 survey data. The Fermi-LAT Collaboration acknowledges generous ongoing support from a number of agencies and institutes that have supported both the development and the operation of the LAT as well as scientific data analysis. These include the National Aeronautics and Space Administration and the Department of Energy in the United States, the Commissariat à l'Energie Atomique and the Centre National de la Recherche Scientifique/Institut National de Physique Nucléaire et de Physique des Particules in France, the Agenzia Spaziale Italiana and the Istituto Nazionale di Fisica Nucleare in Italy, the Ministry of Education, Culture, Sports, Science and Technology (MEXT), High Energy Accelerator Research Organization (KEK) and Japan Aerospace Exploration Agency (JAXA) in Japan, and the K. A. Wallenberg Foundation, the Swedish Research Council and the Swedish National Space Board in Sweden. Additional support for science analysis during the operations phase is gratefully acknowledged from the Istituto Nazionale di Astrofisica in Italy and the Centre National d'Études Spatiales in France. This work performed in part under DOE Contract DE-AC02-76SF00515. We would like to thank Pablo Saz Parkinson for his helpful comments and suggestions.

\section{References}

Abdo, A. A., Ajello, M., Allafort, A., et al. 2013, ApJS, 208, 17

Alpar, M. A., Cheng, A. F., Ruderman, M. A., \& Shaham, J. 1982, Nature, 300, 728

Atwood, W. B., Abdo, A. A., Ackermann, M., et al. 2009, ApJ, 697, 1071

Bates, S. D., Bailes, M., Bhat, N. D. R., et al. 2011, MNRAS, 416, 2455

Bhattacharyya, B., Roy, J., Ray, P. S., et al. 2013, ApJ, 773, L12

Bisnovatyi-Kogan, G. S., \& Komberg, B. V. 1974, Sov. Astron., 18, 217

Bochenek, C., Ransom, S., \& Demorest, P. 2015, ApJ, 813, L4

Breton, R. P., van Kerkwijk, M. H., Roberts, M. S. E., et al. 2013, ApJ, 769, 108

Bruel, P. 2019, A\&A, 622, A108

Carlberg, R. G., \& Innanen, K. A. 1987, AJ, 94, 666

Cognard, I., Theureau, G., Guillemot, L., et al. 2013, in SF2A-2013: Proceedings

of the Annual meeting of the French Society of Astronomy and Astrophysics, eds. L. Cambresy, F. Martins, E. Nuss, \& A. Palacios, 327

Cognard, I., Freire, P. C. C., Guillemot, L., et al. 2017, ApJ, 844, 128 
Cordes, J. M., \& Lazio, T. J. W. 2002, ArXiv e-prints [arXiv:astro-ph/0207156]

Cromartie, H. T., Camilo, F., Kerr, M., et al. 2016, ApJ, 819, 34

Desvignes, G., Cognard, I., Champion, D., et al. 2013, in Neutron Stars and Pulsars: Challenges and Opportunities After 80 Years, ed. J. van Leeuwen, IAU Symp., 291, 375

Desvignes, G., Caballero, R. N., Lentati, L., et al. 2016, MNRAS, 458, 3341

Eggleton, P. P. 1983, ApJ, 268, 368

Fermi-LAT Collaboration 2019, ArXiv e-prints [arXiv:1902 . 10045]

Freire, P. C. C. 2005, in Binary Radio Pulsars, eds. F. A. Rasio, \& I. H. Stairs, ASP Conf. Ser., 328, 405

Freire, P. C. C. 2013, in Neutron Stars and Pulsars: Challenges and Opportunities After 80 Years, ed. J. van Leeuwen, IAU Symp., 291, 243

Fruchter, A. S., Stinebring, D. R., \& Taylor, J. H. 1988, Nature, 333, 237

Fruchter, A. S., Berman, G., Bower, G., et al. 1990, ApJ, 351, 642

Gentile, P. A., Roberts, M. S. E., McLaughlin, M. A., et al. 2014, ApJ, 783, 69

Guillemot, L., \& Tauris, T. M. 2014, MNRAS, 439, 2033

Guillemot, L., Smith, D. A., Laffon, H., et al. 2016, A\&A, 587, A109

Hobbs, G., Lorimer, D. R., Lyne, A. G., \& Kramer, M. 2005, MNRAS, 360, 974

Hobbs, G. B., Edwards, R. T., \& Manchester, R. N. 2006, MNRAS, 369, 655

Hotan, A. W., van Straten, W., \& Manchester, R. N. 2004, PASA, 21, 302

Hou, X., Smith, D. A., Guillemot, L., et al. 2014, A\&A, 570, A44

Jones, M. L., McLaughlin, M. A., Lam, M. T., et al. 2017, ApJ, 841, 125

Kuijken, K., \& Gilmore, G. 1989, MNRAS, 239, 571

Lange, C., Camilo, F., Wex, N., et al. 2001, MNRAS, 326, 274

Li, D., Lin, F. X., Main, R., et al. 2019, MNRAS, 484, 5723
Main, R., Yang, I.-S., Chan, V., et al. 2018, Nature, 557, 522

Manchester, R. N., Hobbs, G. B., Teoh, A., \& Hobbs, M. 2005, AJ, 129, 1993

Octau, F. 2017, PhD Thesis, Université d'Orléans, France

Octau, F., Desvignes, G., Cognard, I., et al. 2016, in SF2A-2016: Proceedings of the Annual meeting of the French Society of Astronomy and Astrophysics, eds. C. Reylé, J. Richard, L. Cambrésy, et al., 61

Octau, F., Cognard, I., Guillemot, L., et al. 2018, A\&A, 612, A78

Pennucci, T. T., Demorest, P. B., \& Ransom, S. M. 2014, ApJ, 790, 93

Polzin, E. J., Breton, R. P., Clarke, A. O., et al. 2018, MNRAS, 476, 1968

Press, W. H., Teukolsky, S. A., Vetterling, W. T., \& Flannery, B. P. 1992,

Numerical Recipes in Fortran, 2nd edn. (Cambridge: Cambridge University Press)

Ransom, S. M., Eikenberry, S. S., \& Middleditch, J. 2002, AJ, 124, 1788

Ray, P. S., Kerr, M., Parent, D., et al. 2011, ApJS, 194, 17

Ray, P. S., Abdo, A. A., Parent, D., et al. 2012, 2011 Fermi Symposium Proceedings - eConf C110509

Ruderman, M., Shaham, J., \& Tavani, M. 1989, ApJ, 336, 507

Shaifullah, G., Verbiest, J. P. W., Freire, P. C. C., et al. 2016, MNRAS, 462, 1029

Shklovskii, I. S. 1970, Sov. Astron., 13, 562

Smith, D. A., Bruel, P., Cognard, I., et al. 2019, ApJ, 871, 78

Stappers, B. W., Bailes, M., Lyne, A. G., et al. 2001, MNRAS, 321, 576

Thompson, C., Blandford, R. D., Evans, C. R., \& Phinney, E. S. 1994, ApJ, 422, 304

Wadiasingh, Z., Harding, A. K., Venter, C., Böttcher, M., \& Baring, M. G. 2017, ApJ, 839, 80

Yao, J. M., Manchester, R. N., \& Wang, N. 2017, ApJ, 835, 29 